\title{
Recovery of respiratory function and autonomic diaphragm movement following unilateral recurrent laryngeal nerve to phrenic nerve anastomosis in rabbits
}

\author{
*Junxiang Wen, MD, PhD, ${ }^{1-3}$ Yingchao Han, MD, PhD, ${ }^{3}$ Song Guo, MD, PhD, ${ }^{3}$ Mingjie Yang, MD, \\ PhD, ${ }^{3}$ Lijun Li, MD, PhD, ${ }^{3}$ Guixin Sun, MD, PhD, ${ }^{4}$ Jun Wang, MD, ${ }^{2}$ Fangqiong Hu, MSc, ${ }^{2}$ \\ Jing Liang, MSc, ${ }^{2}$ Li Wei, BSc, ${ }^{2}$ Qi Zhou, BSc, ${ }^{2}$ Weibin Zhang, MD, PhD, ${ }^{1,2}$ and Jun Tan, MD, PhD ${ }^{3}$ \\ 'Department of Orthopaedics, Ruijin Hospital, Shanghai Jiaotong University School of Medicine; ${ }^{2}$ Shanghai Key Laboratory for \\ Bone and Joint Diseases, Shanghai Institute of Orthopedics and Traumatology, Ruijin Hospital, Shanghai Jiaotong University \\ School of Medicine; and Departments of ${ }^{3}$ Spine Surgery and ${ }^{4}$ Traumatology, Shanghai East Hospital, Tongji University School of \\ Medicine, Shanghai, China
}

OBJECTIVE Respiratory dysfunction is the leading cause of mortality following upper cervical spinal cord injury (SCI). The authors' previous study suggested that vagus nerve (VN) and phrenic nerve (PN) anastomosis could partially improve respiratory function in rabbits that had been subjected to $\mathrm{PN}$ transection. As a branch of the $\mathrm{VN}$ and a motor fiberdominated nerve, the recurrent laryngeal nerve (RLN) seems a better choice to anastomose with the PN for respiratory function restoration after upper cervical SCI. This study was designed to determine whether RLN-PN anastomosis could restore the respiratory function after upper cervical SCl in rabbits.

METHODS Twelve male New Zealand rabbits were randomly divided into 3 groups: 1) sham group (no injury), 2) transection group (right RLN and PN were transected), and 3) bridge group (transected right RLN and PN were immediately anastomosed). Spontaneous discharges of the RLN and PN were compared using a bio-signal collection system. RLN and PN cross sections were stained for acetylcholinesterase (AChE), and the numbers of motor fibers were compared. Three months after the initial surgical procedures, the movement of the diaphragm was assessed using a digital subtraction angiography (DSA) system, and discharges from the right diaphragm muscle were recorded. Toluidine blue staining, electron microscopy, and staining for AChE were used to assess whether motor fibers from the RLN regenerated into the $\mathrm{PN}$, and sections of diaphragm were examined after AChE staining to assess the motor endplates.

RESULTS Both the RLN and PN exhibited highly rhythmic discharges, synchronized with respiration, and most fibers in the RLN and PN were found to be motor fibers. Numerous myelinated fibers were observed in anastomosed PN using toluidine blue staining and electron microscopy. Staining for AChE showed that those regenerated fibers had typical characteristics of motor fibers, and motor endplates with typical morphological characteristics were observed in the diaphragm. Reestablished rhythmic contraction of the hemidiaphragm was directly observed using the DSA system, and rhythmic spontaneous discharge was recorded from the reinnervated hemidiaphragm using the bio-signal collection system.

CONCLUSIONS Motor fibers from the RLN could regenerate into the PN after end-to-end anastomosis and reinnervate the denervated hemidiaphragm in rabbits. Those regenerated motor fibers restored rhythmic and autonomic movement of the paralyzed diaphragm. These results suggest that the RLN is an optimal donor nerve to anastomose with the PN in order to reestablish the autonomic movement of paralyzed diaphragms after high-level SCl.

https://thejns.org/doi/abs/10.3171/2017.12.SPINE17849

KEYWORDS recurrent laryngeal nerve; phrenic nerve; diaphragm; reinnervation; respiration

ABBREVIATIONS AChE = acetylcholinesterase; $D S A=$ digital subtraction angiography; ILN = inferior laryngeal nerve; $P N=$ phrenic nerve; RLN = recurrent laryngeal
nerve; $S A N=$ spinal accessory nerve; $S C I=$ spinal cord injury; $V N=$ vagus nerve.

SUBMITTED August 1, 2017. ACCEPTED December 18, 2017.

INCLUDE WHEN CITING Published online July 6, 2018; DOI: 10.3171/2017.12.SPINE17849.

${ }^{*}$ J. Wen, Y. Han, and S. Guo contributed equally to this work. 
$\mathrm{S}$ PINAL cord injury (SCI) is a life-threatening injury that typically leads to permanent disability or mortality. Approximately $40 \%$ of SCIs occur at the cervical level, ${ }^{23}$ which would cause respiratory function deficits. Anastomosis of a donor nerve with the phrenic nerve $(\mathrm{PN})$ is a potential strategy for reinnervating the diaphragm. Spinal accessory nerve (SAN) and PN anastomosis has been shown to be a possible way to restore the respiratory movement of the diaphragm., ${ }^{9,28,29,31}$ Our previous study showed that vagus nerve (VN) to $\mathrm{PN}$ anastomosis could partially improve respiratory function in rabbits that had been subjected to unilateral PN transection..$^{30}$

Nevertheless, there are drawbacks to using the $\mathrm{VN}$ for anastomosis to the PN, even though the $\mathrm{VN}$ contains some fibers that produce efferent discharges synchronous with the PN. ${ }^{4,13,19}$ First, the total spontaneous efferent discharges of the VN and PN are different in frequency. ${ }^{30}$ Second, more than $80 \%$ of the $\mathrm{VN}$ axons are afferent; ${ }^{10}$ thus, most of the contact area between the VN and PN after anastomosis would be taken up by sensory fibers from the VN. In addition, the $\mathrm{VN}$ is a mixed nerve that branches to viscera and organs, and severing the $\mathrm{VN}$ at the cervical level would cause significant dysfunction. . $^{6,11}$

The recurrent laryngeal nerve (RLN) is a branch of the VN. Baldissera et al. demonstrated that diaphragmatic reinnervation was technically possible through RLN-PN anastomosis. ${ }^{1}$ Tachikawa et al. found that the discharge frequency of the RLN synchronized with that of the PN, and this demonstrated the theoretical possibility of reestablishing autonomic and physiological respiration with RLN-PN anastomosis. ${ }^{26}$ Gauthier et al. found that spontaneous discharge from an anastomosed PN could be detected even after complete transection of the spinal cord at the $\mathrm{C} 2$ level in a rat model. ${ }^{12}$ Using a rabbit model, Derrey et al. studied the effect of anastomosis of the inferior laryngeal nerve (ILN) to the PN and found poor neurogenic electrical activity of the diaphragm. ${ }^{8}$ Furthermore, respiratory function was not assessed after anastomosis in these animal studies, and the effectiveness of respiratory recovery thus remained unknown.

In the present study, we first compared spontaneous discharges and numbers of motor fibers between the rabbit RLN and PN. We then investigated the effectiveness of RLN-PN anastomosis in restoration of autonomic respiration in rabbits that had been subjected to unilateral phrenicotomy by observing contraction of the hemidiaphragm and recording hemidiaphragmatic discharge. Finally, we explored the morphological changes of anastomosed PN using electron microscopy, acetylcholinesterase (AChE) staining, and toluidine blue staining.

\section{Methods}

All animal experiments were conducted in accordance with protocols approved by the Institutional Animal Care Committee of Tongji University.

\section{Animals and Groups}

A total of 12 male New Zealand rabbits ( 3 months old) were included in this study. The rabbits were obtained from the Animal Center of Tongji University, Shanghai,
China, and were maintained at $22^{\circ} \mathrm{C}$ on a 12 -hour light/ dark cycle with free access to rabbit chow and water. To exclude sex differences in respiratory flow and tidal volume, only male animals were used, as in our previous study. ${ }^{30}$ The rabbits were randomly divided into 3 equal groups (Fig. 1): a sham group (4 rabbits) in which both the right RLN and right PN were exposed but not transected, a transection group (4 rabbits) in which the right RLN and $\mathrm{PN}$ were both transected $1.5 \mathrm{~cm}$ rostral to the clavicle, and a bridge group (4 rabbits) in which the right RLN and PN were transected at $1.5 \mathrm{~cm}$ rostral to the clavicle and the caudal cut end of the RLN was immediately anastomosed to the caudal cut end of the PN.

In order to reduce animal use, the 4 animals in the transection group were also used for comparison of spontaneous efferent discharge and motor fiber number between the recurrent laryngeal nerve (RLN) and phrenic nerve (PN). Testing for spontaneous efferent discharge was performed before the transection of RLN and PN. A 1-cm-long nerve segment of the RLN and a same-length segment of the PN were harvested during RLN and PN transection, and these nerve segments were used for study of motor fibers.

\section{Initial Surgical Procedures}

The anesthesia and surgical procedures were similar to those used in our previous study..$^{30}$ The animals were anesthetized with $2 \%$ pentobarbital $(1.5 \mathrm{ml} / \mathrm{kg})$ injected through the marginal ear vein. The surgery was performed via a ventral medial approach, with the animals in a supine position. The RLN was exposed in the tracheoesophageal fascia running between the trachea and the esophagus. The PN was exposed on the surface of the anterior scalene muscle. In the sham (control) group, both the RLN and PN were exposed but left intact. In the transection group, the right RLN and PN were transected at a level $1.5 \mathrm{~cm}$ higher than the clavicle and a $10-\mathrm{mm}$ segment of each nerve was removed to prevent regeneration. These specimens were used for the study of motor fibers (see below). In the bridge group, the RLN and PN were transected $1.5 \mathrm{~cm}$ above the clavicle and the caudal cut end of the RLN was anastomosed to the caudal cut end of the PN by epineurial suturing with 10.0 nylon thread (Alcon) under a surgical microscope (SXP-1B, Medical Optical Company). The incision was cleaned with iodine and saline and then closed without drain placement. All animals received postoperative analgesic treatment for 3 days (buprenorphine, $0.02 \mathrm{mg} / \mathrm{kg}$, subcutaneous injection, Yangzhou No. 3 Pharmaceutical Co., Ltd.).

\section{Spontaneous Efferent Discharges From RLN and PN}

Spontaneous efferent discharges were recorded from the RLN and PN in the 4 rabbits in the transection group before the procedure described above but during the same surgical session. These 4 rabbits were initially anesthetized using $20 \%$ urethane $(4 \mathrm{ml} / \mathrm{kg})$, and both RLN and PN were carefully exposed under a surgical microscope (SXP1B). Nerve discharges were recorded using the RM6240 bio-signal collection system (Chengdu Instrument Plant). Briefly, the RLN or PN was well dissected and placed on a bipolar metal recording electrode (YSD100, Chengdu In- 
strument Plant). Spontaneous discharges were band-pass filtered (200 to $5000 \mathrm{~Hz}$ ), amplified (8000x), recorded, and analyzed using RM6240 bio-signal analysis software (Chengdu Instrument Plant). Respiratory waves were also recorded simultaneously with efferent discharge by means of a noninvasive respiratory recorder (HX100, Chengdu Instrument Plant).

\section{Study of Motor Fibers}

AChE staining was performed on cross sections of PN and RLN, and the numbers of motor fibers in the PN and RLN were calculated (see below).

\section{Diaphragm Movement Observation}

Twelve weeks after surgery, the rabbits were anesthetized using $20 \%$ urethane $(4 \mathrm{ml} / \mathrm{kg}$ ) and fixed to a wooden board in a supine position under a digital subtraction angiography (DSA) system (Allura Xper FD-20, Philips) to record anterior-posterior video of the diaphragm movement. The movement of the diaphragm was calculated by measuring the vertical displacement of the middle point of the left or right diaphragm. The measurement and analysis were performed by experimenters (M.Y. and L.L.) blind to the source video.

\section{Diaphragm Muscle Discharges}

With the animals still in a supine position, laparotomy was performed, and the liver was gently separated and pushed away to expose the diaphragm muscle. The discharge activity of the right diaphragm was investigated. Compound muscular activity was recorded by inserting a bipolar copper needle-shaped recording electrode into the center of the right hemidiaphragm. The signals were filtered $(0.5-3 \mathrm{kHz})$, amplified, and displayed as the diaphragm electromyogram. The diaphragm electromyographic activity was recorded during spontaneous breathing. Recorded data were saved and analyzed using RM6240 bio-signal analysis software (Chengdu Instrument Plant).

\section{Tissue Harvest}

After recording of diaphragm muscle discharges, the animals in all 3 groups were killed by injecting air into the marginal ear vein.

The RLN and PN were harvested from the animals in the sham group. The PN was harvested from animals in the transection group and the bridge group. The RLN was harvested from the neck area, and the PN was harvested from the thoracic area. Harvested RLN and PN specimens were prepared for AChE staining, toluidine blue staining, and transmission electron microscopy observation.

Right diaphragm muscles were harvested from animals in all 3 groups for AChE staining.

\section{Acetylcholinesterase Staining}

Harvested nerve segments and diaphragm specimens were fixed in $10 \%$ formalin for $48-72$ hours at $4^{\circ} \mathrm{C}$, transferred to $30 \%$ sucrose and maintained at $4{ }^{\circ} \mathrm{C}$ for an additional $24-48$ hours, and then embedded in optimal cutting
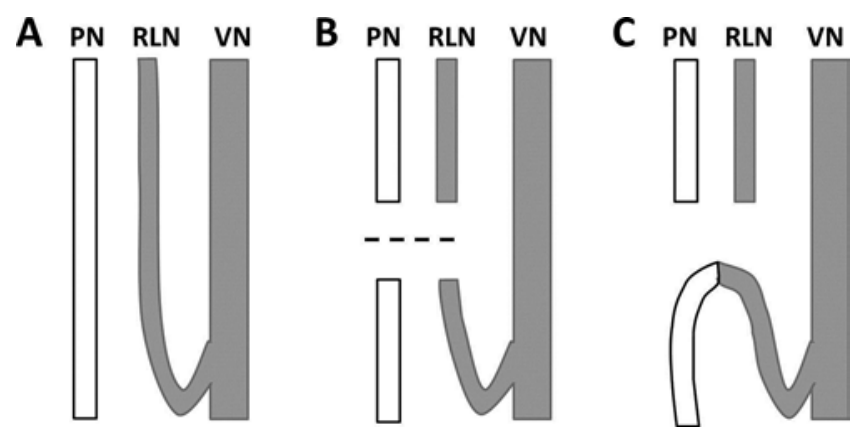

FIG. 1. Schematic showing the initial surgical procedures in the 3 groups. A: Sham group. Both the PN and RLN were left intact. B: Transection group. Both the PN and RLN were transected. C: Bridge group. The caudal cut end of the RLN was anastomosed to the caudal cut end of the PN. PN = phrenic nerve; RLN = recurrent laryngeal nerve; $V N=$ vagus nerve.

temperature (OCT) compound (Fisher Scientific). Frozen sections of 10- $\mu \mathrm{m}$ thickness were cut on a freezing microtome (Bright OTF5000, Scilogex), and every fifth section was collected and mounted on a slide. Slides were heated to $37^{\circ} \mathrm{C}$ for 30 minutes and then stored at $-80^{\circ} \mathrm{C}$ until they were stained for AChE. AChE staining was performed according to the method of Karnovsky and Roots. ${ }^{17}$ Briefly, sections were immersed in an incubation medium consisting of iodized acetylthiocholine, potassium ferricyanide, and copper sulfate. Iodized acetylthiocholine acts as a substrate for $\mathrm{AChE}$, and the latter 2 chemicals react with the liberated thiocholine, producing a brownish precipitate. After incubation for 6 hours at room temperature, the slides were lightly counterstained with Harris hematoxylin. Sections were examined using an imaging microscope (Carl Zeiss) and analyzed with ImageJ (NIH) by experimenters (M.Y. and L.L.) blind to the source nerve and diaphragm to determine the numbers of motor fibers and motor endplates, respectively.

\section{Toluidine Blue Staining}

Harvested nerve segments were fixed at $4^{\circ} \mathrm{C}$ with $3 \%$ glutaraldehyde (Polysciences Inc.), washed in 0.1-M phosphate buffer, postfixed with $1 \%$ osmium tetroxide (Fisher Scientific), dehydrated in graded ethanol solutions, and embedded in Araldite 502 (Polysciences Inc.). Semi-thin sections $(1 \mu \mathrm{m}$ thick) were stained with $1 \%$ toluidine blue for histomorphometric analysis. Sections were examined using an imaging microscope (Carl Zeiss) and analyzed with ImageJ by experimenters (M.Y. and L.L.) blind to the source nerve to determine the numbers of myelinated fibers and area of total myelinated fibers.

\section{Nerve Ultrastructure Visualization by Transmission Electron Microscopy}

Nerve specimens were fixed with precooled $2.5 \%$ glutaraldehyde (Polysciences Inc.) in 0.1-M phosphatebuffered saline for 1-2 hours and postfixed in 1\% osmium tetraoxide solution (Fisher Scientific) for 1 hour. Then they were dehydrated stepwise in increasing concentrations of ethanol and embedded in Epon 812 epoxy resin. The specimens were cut into ultra-thin sections, $70 \mathrm{~nm}$ thick, 

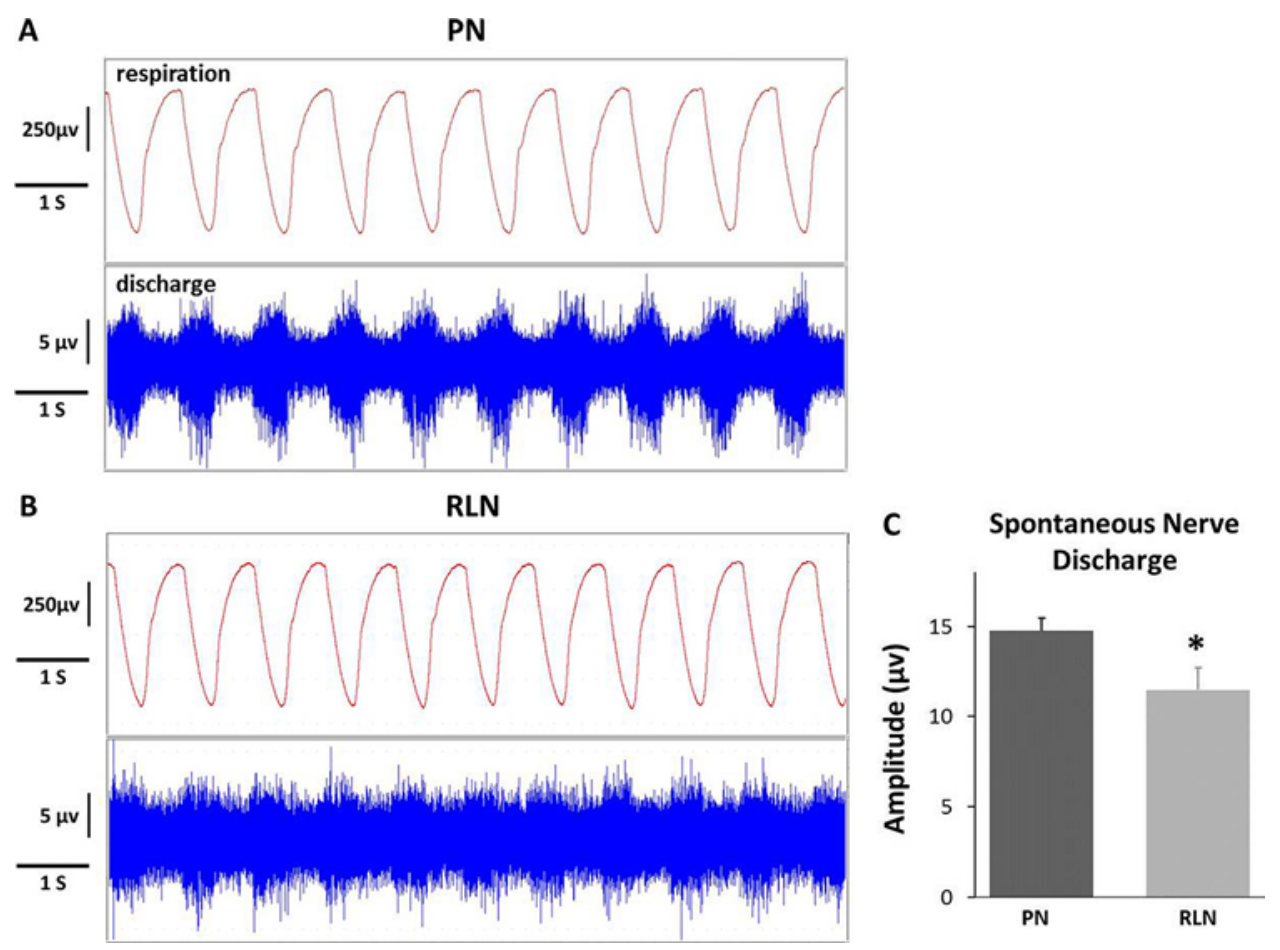

FIG. 2. Spontaneous discharges from the PN and RLN. A: Rhythmic discharges from the PN. The upper row shows a recording of respiration. The lower row shows the rhythmic simultaneous recording of the PN discharge. The discharge frequency of the PN was highly rhythmic and consistent with the respiration frequency. B: Rhythmic discharges from the RLN. The upper row shows the recording of respiration. The lower row shows the rhythmic simultaneous recording of the RLN discharge. The discharge frequency of the RLN was highly rhythmic and consistent with the respiration frequency. C: Discharge amplitudes of the PN and RLN $(p=0.003)$. The error bars indicate standard deviations. ${ }^{*} p<0.01 . S=$ seconds. Figure is available in color online only.

stained with lead citrate and uranyl acetate, and examined by transmission electron microscopy (H-7650, Hitachi). The electron microscopy images from 5 random fields of each nerve section were selected and analyzed with ImageJ by experimenters (M.Y. and L.L.) blind to the source nerve to determine the thickness of the myelin sheath.

\section{Statistical Analysis}

All results are expressed as mean \pm standard deviation. PN and RLN parameters were compared by 1-way analysis of variance (ANOVA). Multiple comparisons among the 3 groups were evaluated using Scheffé's test (post hoc analysis). A p value $<0.05$ was considered statistically significant. Data were analyzed using SPSS 14.0 software (SPSS Inc.).

\section{Results}

\section{Comparison of Spontaneous Discharges of the PN and RLN}

Spontaneous discharges of the PN and RLN were recorded separately. Respiration frequency was also recorded simultaneously with nerve discharge. The discharge frequencies of the PN (Fig. 2A) and RLN (Fig. 2B) were both highly rhythmic and consistent with the respiration frequency. In addition, individual volleys recorded from the PN and RLN were of a relatively uniform pattern. However, the mean amplitude of PN discharges was high- er than that of RLN discharges $(14.76 \pm 0.69 \mu \mathrm{V}$ vs 11.48 $\pm 1.23 \mu \mathrm{V}, \mathrm{p}<0.01)$.

\section{Diaphragm Movement}

Diaphragm movements were recorded using a DSA system (see Fig. 7). Measurement of the diaphragm movement was performed by comparing the vertical displacement of the midpoint of either side of the diaphragm. The movement of the diaphragm was defined as the displacement between the end of inspiration and the end of expiration. The movement measurements for the left diaphragm (no-surgery side) were $0.516 \pm 0.080 \mathrm{~cm}$ for the sham group, $0.650 \pm 0.080 \mathrm{~cm}$ for the transection group, and $0.633 \pm 0.076 \mathrm{~cm}$ for the bridge group. The measurements for the right diaphragm (surgery side) were $0.523 \pm 0.060$ $\mathrm{cm}$ for the sham group, $0.073 \pm 0.030 \mathrm{~cm}$ for the transection group, and $0.505 \pm 0.124 \mathrm{~cm}$ for the bridge group.

There was no statistically significant difference in the movement of the left diaphragm (no-surgery side) among the 3 groups $(p>0.05)$. On the right side, there was significantly less diaphragm movement in the transection group than in the sham group $(\mathrm{p}<0.001)$ or the bridge group $(\mathrm{p}<0.001)$. However, there was no significant difference between the sham group and the bridge group $(p>0.05)$. In the bridge group, there was a significant difference between the diaphragm movement on the left side and that on the right side $(\mathrm{p}<0.001)$. 


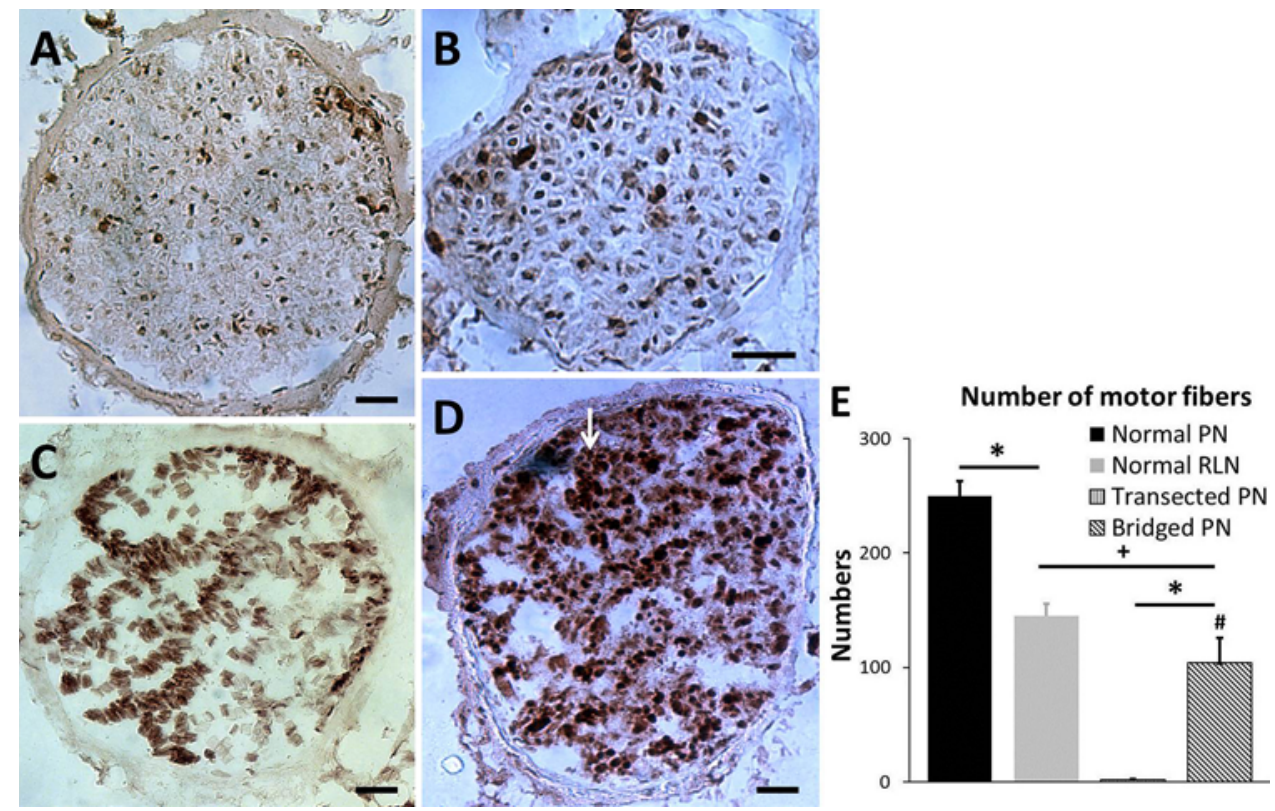

FIG. 3. AChE staining of normal PN, normal RLN, transected PN, and bridged PN. A: Normal PN. Almost all fibers presented the typical characteristics of motor fibers, with AChE-stained axons and unstained myelin sheaths. B: Normal RLN. Almost all fibers presented the typical characteristics of motor fibers. C: Transected PN. All fibers were degenerated, and no normal staining of neural fiber was shown. D: PN bridged with RLN. Some fibers showed typical characteristics of motor fibers (arrow). Scale bar = $25 \mu \mathrm{m}(\mathrm{A}-\mathrm{D})$. E: Comparison of motor fiber numbers. The error bars indicate standard deviations. ${ }^{*} p<0.0001,+p=0.009$, \#p< 0.0001 versus normal PN group. Figure is available in color online only.

\section{Spontaneous Discharges of the Right Diaphragm}

The discharges of the right diaphragm were investigated in the 3 groups. Regular and rhythmic discharge waves were seen in the sham group (see Fig. 8A), but no apparent discharge wave was detected in the transection group (see Fig. 8B). However, in the bridge group, regular and rhythmic discharge waves were observed 3 months after RLN-PN anastomosis (see Fig. 8C), but those waves were shorter and thinner than those recorded in the sham group. The amplitudes of discharge were $520.33 \pm 57.59 \mu \mathrm{V}$ for the sham group, $20.25 \pm 10.69 \mu \mathrm{V}$ for the transection group, and $403.75 \pm 76.05 \mu \mathrm{V}$ for the bridge group. There were statistically significant differences between the sham group and the transection group $(\mathrm{p}<0.0001)$ and between the bridge group and the transection group $(p<0.0001)$. However, there was no significant difference between the sham group and the bridge group $(\mathrm{p}=0.068)$.

\section{Numbers of Motor Fibers in the PN and RLN}

Acetylcholinesterase staining was performed on cross sections of PN and RLN, and the numbers of motor fibers in the PN and RLN were calculated. Almost all fibers of the PN (Fig. 3A) and RLN (Fig. 3B) demonstrated the typical morphological and histochemical properties of motor fibers; the axons were stained brown and the myelin sheaths were unstained.

In the transection group, 3 months after the PN was severed (Fig. 3C) all structures in the PN were degenerated and no typical motor fibers were seen. However, when the PN was anastomosed with the RLN (bridge group, Fig. $3 \mathrm{D})$, structures in the PN did not degenerate like those in the PN of the transection group, and typical motor fibers were observed again in the PN (arrow in Fig. 3D). Twenty images from each nerve specimen were analyzed using ImageJ by researchers (M.Y. and L.L.) blind to nerve identity to estimate the number of motor fibers, similar to our previous study. ${ }^{30}$ There were significant differences between the numbers of motor fibers in the normal PN and the normal RLN $(249.75 \pm 12.89$ vs $145.25 \pm 10.53$, p $<0.0001)$, the transected PN and the bridged PN $(2.00 \pm$ 0.82 vs $104.50 \pm 21.06, p<0.0001)$, the normal $P N$ and the bridged PN $(249.75 \pm 12.89$ vs $104.50 \pm 21.06, p<0.0001)$, and the normal RLN and the bridged PN (145.25 \pm 10.53 vs $104.50 \pm 21.06, \mathrm{p}=0.009$ ).

\section{Numbers and Areas of Endplates in the Right Diaphragm}

Sections from the right diaphragm were stained for $\mathrm{AChE}$, and the numbers and areas of endplates were calculated (Fig. 4). In the sham group, the typical endplate morphology, a round shape and brown after staining for AChE (Fig. 4A), was seen in the diaphragm. In contrast, in sections from animals in the transection group (in which the PN was severed), no endplates were seen in the right diaphragm (Fig. 4B). However, in the animals in which the PN had been anastomosed with the RLN (Fig. 4C), endplates could again be observed in the right diaphragm. Twenty images from each diaphragm specimen were analyzed using ImageJ by researchers (M.Y. and L.L.) blind to diaphragm identity to estimate the number and area of endplates. There were significant differences between the numbers of endplates in the sham group and the transection group (17.33 \pm 2.52 vs $0.50 \pm 0.58, \mathrm{p}=0.0002)$, 

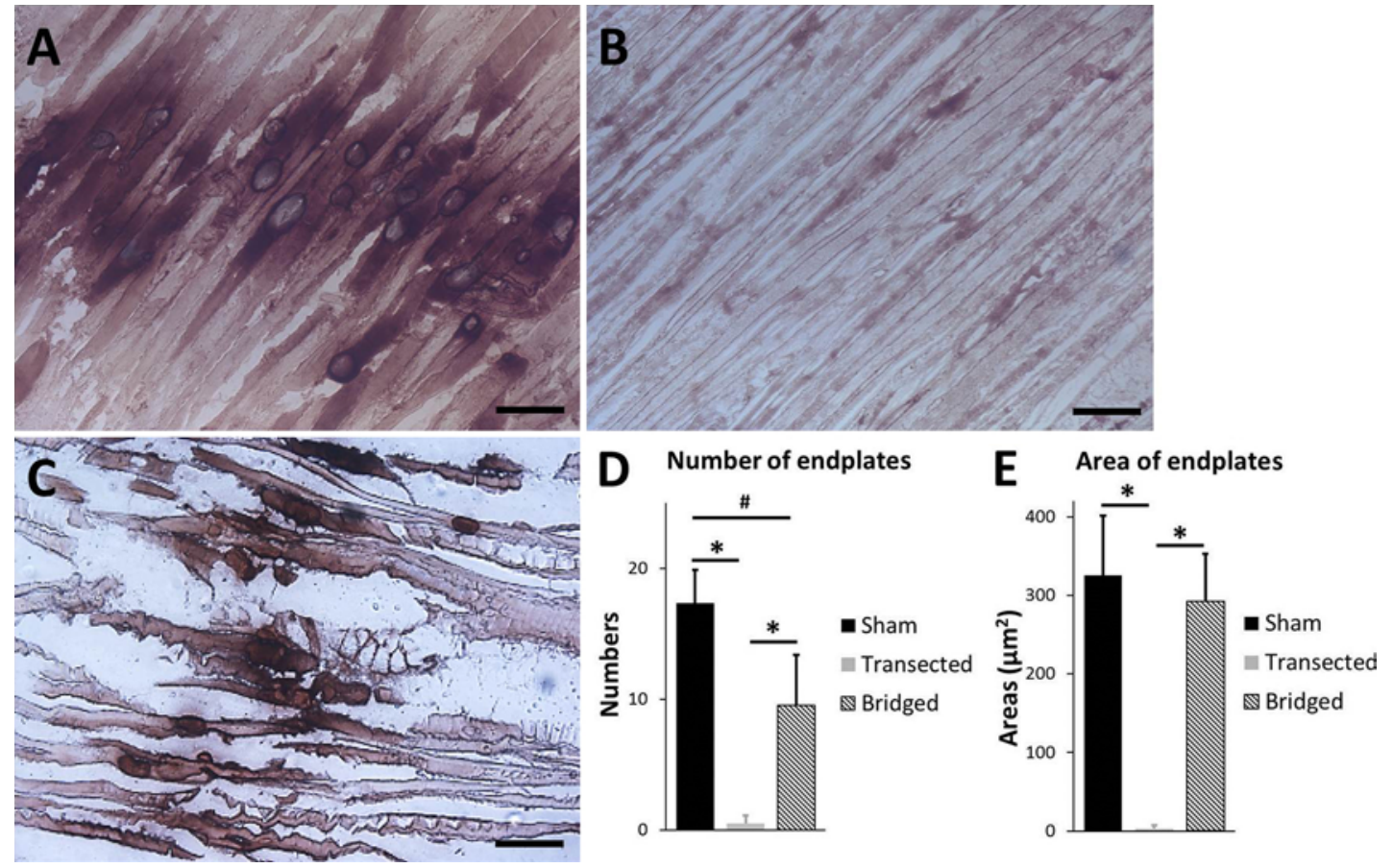

FIG. 4. AChE staining of right diaphragm muscle from the sham group, transection group, and bridge group. A: Sham group. AChE-stained round endplate (as shown here) was visible. B: Transection group. No endplate was observed. C: Bridge group. AChE-stained round endplate was observed, but the morphology was not as typical as that in the sham group. Scale bar $=100 \mu m$ $(A-C)$. D: Comparison of numbers of endplates. E: Comparison of endplate areas. The error bars indicate standard deviations. ${ }^{*} p<0.01, \# p<0.05$. Figure is available in color online only.

the transection group and the bridge group $(0.50 \pm 0.58$ vs $9.50 \pm 3.87, \mathrm{p}=0.0064)$, and the sham group and the bridge group $(17.33 \pm 2.52$ vs $9.50 \pm 3.87, \mathrm{p}=0.0177)$. To obtain an estimate of the area of a single endplate, 5 random endplates in each image were measured. There were significant differences in area between the sham group and the transection group $\left(325.33 \pm 76.25 \mu \mathrm{m}^{2}\right.$ vs $3.50 \pm$ $4.12 \mu \mathrm{m}^{2}, \mathrm{p}=0.0002$ ) and between the transection group and the bridge group $\left(3.50 \pm 4.12 \mu \mathrm{m}^{2}\right.$ vs $292.25 \pm 60.20$ $\mu \mathrm{m}^{2}, \mathrm{p}=0.0002$ ), but not between the sham group and the bridge group $\left(325.33 \pm 76.25 \mu \mathrm{m}^{2}\right.$ vs $292.25 \pm 60.20 \mu \mathrm{m}^{2}$, $\mathrm{p}=0.7291)$.

\section{Number and Area of Myelinated Fibers}

PN and RLN cross sections were stained with toluidine blue, and the number of myelinated fibers and area of total myelinated fibers were calculated. Normal PN and RLN were filled with myelinated fibers (Fig. 5A and B). In specimens from the transection group, 3 months after the PN was severed, no myelin sheath was noticed (Fig. 5C). However, myelinated fibers were observed when the PN was anastomosed with the RLN (bridge group, Fig. 5D), although those fibers appeared, based on visual inspection, to vary substantially in diameter. The numbers of myelinated fibers were $405.50 \pm 18.34$ for normal PN, $199.75 \pm 11.03$ for normal RLN, $0 \pm 0$ for transected PN, and $292.75 \pm 39.69$ for bridged PN. There were significant differences in the number of myelinated fibers between the normal PN and normal RLN ( $<<0.0001)$, between the bridged PN and the transected PN $(\mathrm{p}<0.0001)$, between the bridged PN and the normal PN ( $<<0.0001)$, and between the bridged PN and the normal RLN ( $p=0.0002)$.

The mean area of total myelinated fibers was 20,954.43 $\pm 2647.62 \mu \mathrm{m}^{2}$ for normal PN, 11,931.00 $\pm 1449.81 \mu \mathrm{m}^{2}$ for normal RLN, $0 \pm 0 \mu \mathrm{m}^{2}$ for transected PN, and $4941.58 \pm$ $937.37 \mu \mathrm{m}^{2}$ for bridged PN. Comparison of these values showed statistically significant differences between the normal PN and normal RLN ( $p<0.0001$ ), between the bridged PN and the transected PN $(\mathrm{p}<0.0001)$, between the bridged PN and the normal PN ( $<<0.0001)$, and between the bridged PN and the normal RLN ( $<<0.0001)$.

\section{Myelin Sheath Thickness}

Ultrastructure observations of cross sections were performed under transmission electron microscopy, and the thickness of the myelin sheath was calculated (Fig. 6). Sections of normal PN (Fig. 6A) showed the thickest myelin sheaths $(1.81 \pm 0.16 \mu \mathrm{m})$, with all myelin sheaths of similar thickness. The myelin sheaths in the normal RLN (Fig. 6B) were much thinner $(0.50 \pm 0.23 \mu \mathrm{m})$, and there was much more variability in thickness than in the other groups. In sections of PN obtained 3 months after the nerve was severed, no myelinated fiber was observed, but there were degenerated fibers (Fig. 6C), which looked like onion skin. When the PN was anastomosed with the RLN, some myelinated fibers could be observed in the bridged PN (Fig. 6D). But the myelin sheaths in bridged PN (0.32 $\pm 0.10 \mu \mathrm{m}$ ) were thinner than those in normal PN and normal RLN, and some degenerated myelin sheath could also be seen. 

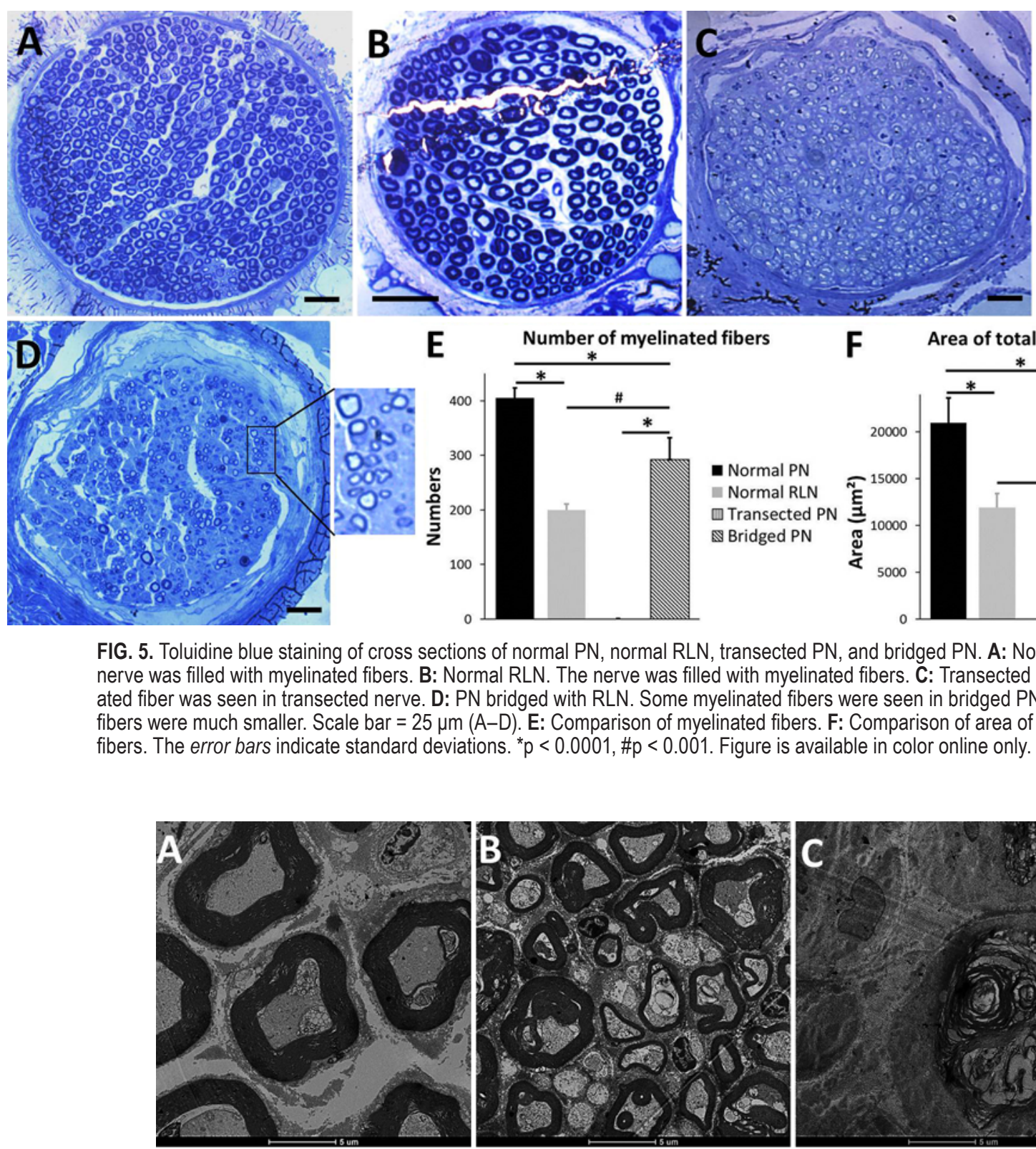

FIG. 5. Toluidine blue staining of cross sections of normal PN, normal RLN, transected PN, and bridged PN. A: Normal PN. The nerve was filled with myelinated fibers. B: Normal RLN. The nerve was filled with myelinated fibers. C: Transected PN. No myelinated fiber was seen in transected nerve. D: PN bridged with RLN. Some myelinated fibers were seen in bridged PN, but most fibers were much smaller. Scale bar $=25 \mu \mathrm{m}(\mathrm{A}-\mathrm{D})$. E: Comparison of myelinated fibers. F: Comparison of area of total myelinated fibers. The error bars indicate standard deviations. ${ }^{*} p<0.0001$, \#p $<0.001$. Figure is available in color online only.
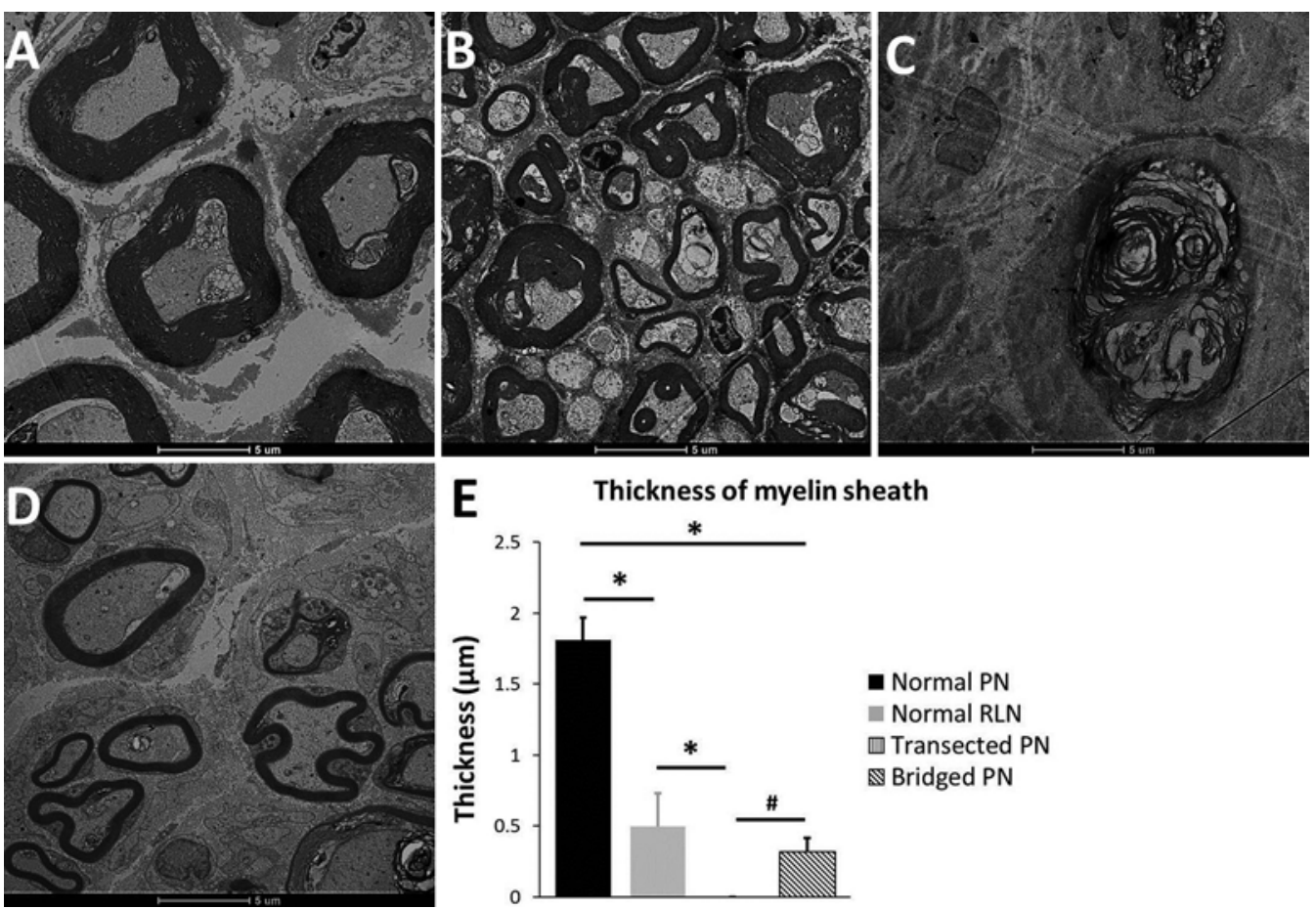

FIG. 6. Ultrastructure observation under transmission electron microscopy of cross section of the normal PN, normal RLN, transected PN, and bridged PN. A: Normal PN. The nerve was filled with myelinated fibers, and the myelin sheaths were similar in thickness. B: Normal RLN. The nerve also was filled with myelinated fibers, but the thickness of the myelin sheaths was not as consistent as in normal PN. C: Transected PN. No myelinated fiber was seen in transected nerve; degenerated fibers were seen. D: PN bridged with RLN. Some myelinated fibers were seen in bridged PN, but the myelin sheaths were thinner than in normal PN and normal RLN. Scale bar $=5 \mu \mathrm{m}(\mathrm{A}-\mathrm{D})$. E: Comparison of myelin sheath thickness. The error bars indicate standard deviations. ${ }^{*} p<0.001, \# p<0.05$. 
Twenty images from each nerve specimen were analyzed using ImageJ by researchers (M.Y. and L.L.) blind to nerve identity to estimate myelin sheath thickness. There were significant differences between the normal PN and each of the other groups ( $<<0.0001)$, between the normal RLN and the transected PN ( $p=0.0009)$, and between the bridged PN and the transected PN ( $p=0.0316)$. However, no significant difference was observed between the normal RLN and the bridged PN ( $\mathrm{p}=0.3370)$.

\section{Discussion}

Respiratory function deficit is a devastating consequence of high-level SCI since there is still no effective method to repair injured neuron cells. Mechanical ventilation remains the most common and effective method of managing this condition. However, mechanical ventilation is associated with extremely low quality of life and serious complications, such as pneumonia and atrophy of the diaphragm.

Restoring the function of the phrenic nerve (PN) through a donor nerve might be a potential strategy to restore contraction of the diaphragm. The spinal accessory nerve (SAN) has been shown to be a possible donor nerve for anastomosis with the PN to restore physiological respiration. ${ }^{9,28,29,31}$ However, the SAN does not have spontaneous discharge, and an external electronic pacing has to be provided. Spontaneous discharge from a donor nerve is important for restoration of spontaneous respiratory function. Reinnervation of the cat diaphragm with spinal accessory, facial, or long thoracic nerves did not improve respiratory function, ${ }^{9}$ because these nerves do not discharge spontaneously. ${ }^{13}$ Our previous study showed that vagus nerve (VN)-PN anastomosis could partially improve respiratory function in rabbits that had undergone unilateral phrenicotomy ${ }^{30}$ In rats, Guth and Frank found that fibers from the $\mathrm{VN}$ regenerated into the PN after VN-PN anastomosis and innervated the diaphragm. ${ }^{13}$ Contraction of the previously paralyzed diaphragm was observed after $\mathrm{VN}$ $\mathrm{PN}$ anastomosis in rats and dogs ${ }^{3,13}$ However, the $\mathrm{VN}$ is not an optimal candidate for anastomosis to the PN because of differing frequencies of their spontaneous discharges, ${ }^{30}$ unsatisfied efficiency of motor fiber regeneration from the $\mathrm{VN}$ into the $\mathrm{PN},{ }^{10,30}$ and interruption of homeostasis after vagotomy at the cervical level. ${ }^{6,11}$

The recurrent laryngeal nerve (RLN) is a branch of the VN. As an important motor nerve of the laryngeal area, the RLN mainly innervates the laryngeal abductors (posterior cricoarytenoid muscle) and laryngeal adductors (lateral cricoarytenoid muscle and thyroarytenoid muscle). ${ }^{18}$ In the present study, we demonstrated that although the amplitude of discharge from the RLN was lower than that of the $\mathrm{PN}$, the spontaneous discharge of the RLN synchronized with that of the PN (Fig. 2). Similarly, Tachikawa et al. found that the discharge frequency of the RLN synchronized with that of the PN whether in a normocapnic condition or hypercapnic acidosis, ${ }^{26}$ and other studies found that RLN-innervated muscles contracted simultaneously with the abdominal muscles during forced expiration, such as when coughing. ${ }^{7,24}$ In addition, we showed that most of the fibers in the RLN are motor fibers using AChE staining, even though the number of motor fibers in the RLN was much less than that in the PN (Fig. 3). Research has shown that the greatest number of regenerated myelinated axons is obtained by anastomosing a proximal donor nerve with fewer axons to a distal nerve stump..$^{15}$ Accordingly, anastomosis of the RLN, which has fewer myelinated axons, to the PN should result in a high number of regenerated myelinated axons. The above findings suggest that neurotization of the PN using the RLN to restore autonomic respiration after high-level SCI is theoretically feasible.

We then found that fibers from the RLN could regenerate into the PN through end-to-end anastomosis in a rabbit model. Using AChE staining, we found that all fibers in the transected PN were degenerated (Fig. 3C), while regenerated fibers in the PN from the animals that had undergone RLN-PN anastomosis demonstrated typical characteristics of motor neurons, which have AChE-stained axons and unstained myelin sheaths (Fig. 3D). Studies have suggested that the fibers that regenerate from donor nerve into recipient nerve are likely nonselective; ${ }^{2,13}$ thus, if one uses a nerve in which most fibers are motor fibers to anastomose with the PN, most regenerated fibers in the PN should be motor fibers. In the present study, we directly demonstrated that motor fibers from the RLN could regenerate into the PN through end-to-end anastomosis. This is of great importance, since we have seen that motor fibers from a donor nerve, which synchronized its spontaneous discharge with respiration frequency, regenerated into the $\mathrm{PN}$, providing the theoretical potential to reestablish autonomic respiration after high-level SCI.

We also demonstrated the change of myelin sheath in anastomosed PN, since myelin sheath is important in electronic signal transmission. ${ }^{21}$ Using toluidine blue staining and electron microscopy, we found regenerated myelinated fibers in the anastomosed PN (Figs. 5D and 6D). The number of myelinated fibers in anastomosed PN was even more than that in the RLN (Fig. 5E), while the area of myelinated fibers was less than that in the RLN (Fig. $5 \mathrm{~F}$ ), and the thickness of the myelin sheath did not differ significantly between the RLN and anastomosed PN (Fig. $6 \mathrm{E})$. Other studies also found that the number of regenerated fibers in the distal stump was greater than that in the donor nerve and the thickness of the myelin sheath did not change much. ${ }^{15,20}$ Researchers have suggested that the reason for this phenomenon may be that sprouting is in the nature of a peripheral nerve and that axons may sprout and send out collaterals when the integrity of the peripheral nerve is broken..$^{5,20}$

Furthermore, using AChE staining, we demonstrated restoration of diaphragm endplates with RLN-PN anastomosis (Fig. 4). This is direct evidence that anastomosed PN reinnervated the denervated hemidiaphragm, because the motor endplate is a chemical synapse formed by the contact between a motor nerve fiber and a muscle fiber. ${ }^{22}$ Thus, in the present study, we showed morphologically that motor fibers from the RLN could regenerate into the PN through end-to-end anastomosis and reinnervate the denervated hemidiaphragm.

To explore whether the motor fibers in the PN that regenerated from the RLN restored the rhythmic and autonomic contraction of the denervated hemidiaphragm, we used a DSA system to observe the movement of the 

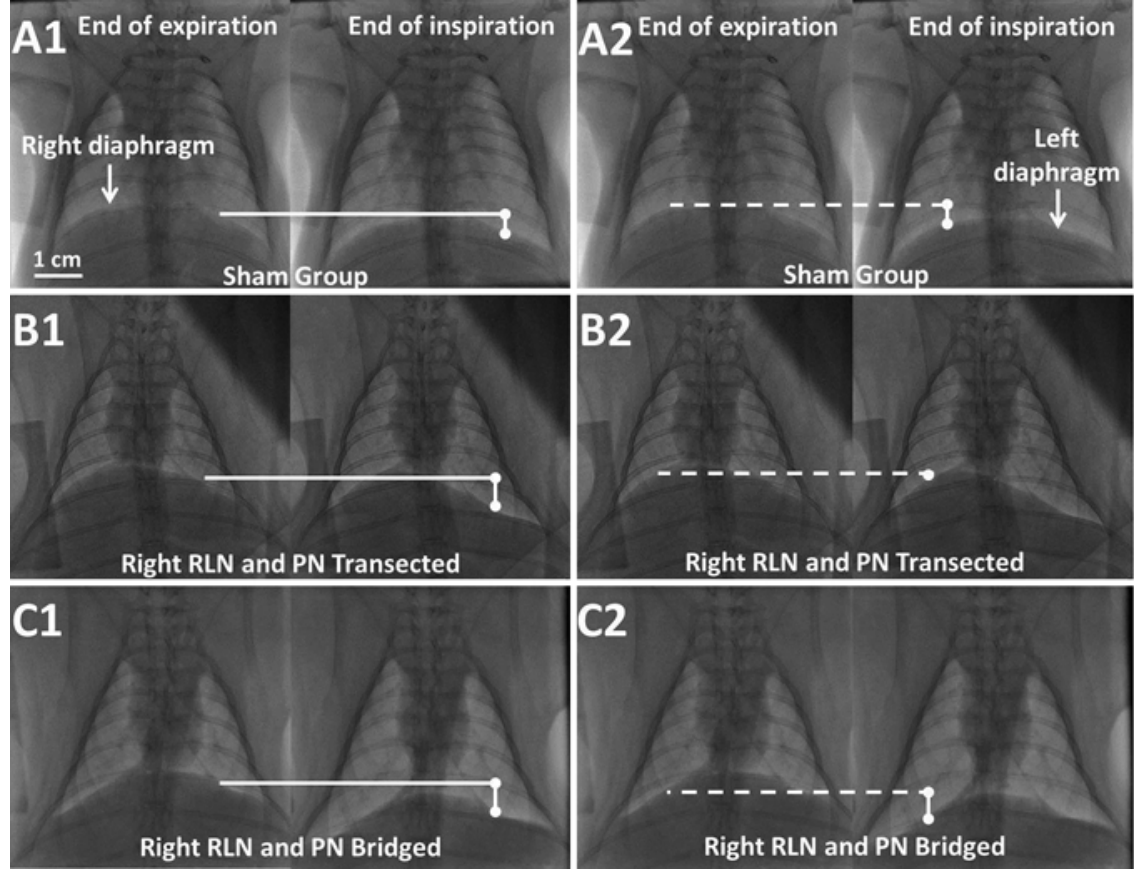

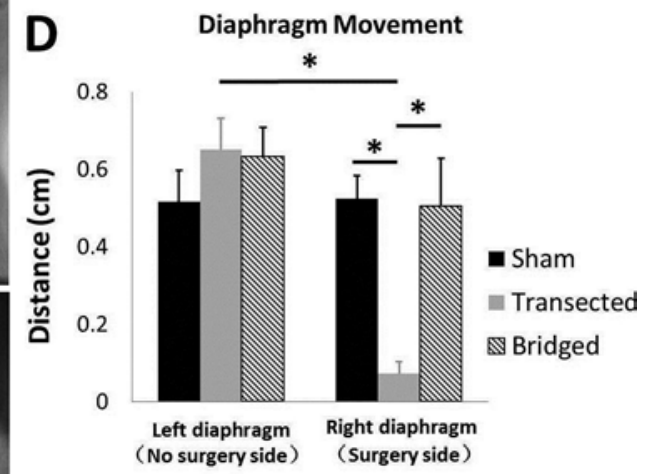

FIG. 7. Movement of diaphragm in the sham group, transection group, and bridge group. In each panel, the image on the left was obtained at the end of expiration and the image on the right was obtained at the end of inspiration. The length of short vertical lines with dots on the ends indicates the displacement of the diaphragm between the end of inspiration and the end of expiration. A1, B1, and C1: Measurement of left diaphragm movement in the sham group, transection group, and bridge group. The middle point of the left diaphragm was selected to calculate the distance of movement. A2, B2, and C2: Measurement of right diaphragm movement in the sham group, transection group, and bridge group. The middle point of the left diaphragm was selected to calculate the distance of movement. D: Comparison of diaphragm movement. The error bars indicate standard deviations. ${ }^{*} p<0.0001$.

hemidiaphragm directly (Fig. 7). Diaphragm movement was barely noticeable on the surgical side in the transection group, whereas on the surgical side in the bridge group, movement had been reestablished and the distance of movement was not significantly different from that of normal diaphragm (Fig. 7). This was the first time that restored rhythmic and autonomic movement of paralyzed diaphragm has been recorded directly. Our results indicate that the RLN is an optimal donor nerve to anastomose with the PN in order to restore the autonomic movement of a paralyzed diaphragm. Diaphragm discharges were also recorded (Fig. 8), and not surprisingly, no discharge was recorded from the denervated diaphragm of the transection group, whereas rhythmic spontaneous discharges were recorded from the reinnervated hemidiaphragm of the bridge group, as in the sham group. Researchers found poor neurogenic electrical inspiratory activity of the diaphragm during quiet breathing after connecting an inferior laryngeal nerve (ILN) to the PN in a rabbit model; however, the electrical activity increased under acute hypoxia. ${ }^{8,25}$ We thought that the RLN would be better than the ILN for anastomosis with the PN to restore function of denervated diaphragm because the RLN contains more motor fibers than the ILN, and thus more fibers would regenerate into the PN and the amplitude of discharge would be higher, which may be of benefit for better response of the reinnervated diaphragm. Gauthier et al. also found that spontaneous discharge from anastomosed PN could be detected even after complete section of the spinal cord at the $\mathrm{C} 2$ level in a rat model when using the RLN as the donor nerve. ${ }^{12}$ However, in their study, respiratory function was not assessed, and the effectiveness of respiratory recovery was unknown. In addition, they used rats to test the feasibility of RLN-PN anastomosis in reestablishing diaphragm autonomy, whereas in the present study we used rabbits, because a larger animal would allow us to observe respiratory function more conveniently and successful application of a new surgical strategy in a larger animal model could give us more confidence and solid theoretic support before we attempt to use it in treating patients.

Although our results demonstrated that RLN-PN anastomosis was able to restore the autonomic contraction of the denervated hemidiaphragm, the function of the RLN was sacrificed, which would result in hoarseness and even dyspnea in human patients because of the absence of movement of the vocal cords on the affected side, ${ }^{16,27,33}$ and bilateral end-to-end anastomosis between the RLN and PN would be impossible. Hence, future studies should attempt anastomosis with the RLN without sacrificing its original function, and end-to-side neurorrhaphy might be a feasible solution. ${ }^{5,14,32}$

\section{Conclusions}

In the present study, we first found that the rabbit RLN and PN both exhibited highly rhythmic discharges that synchronized with respiration and that most fibers in the 


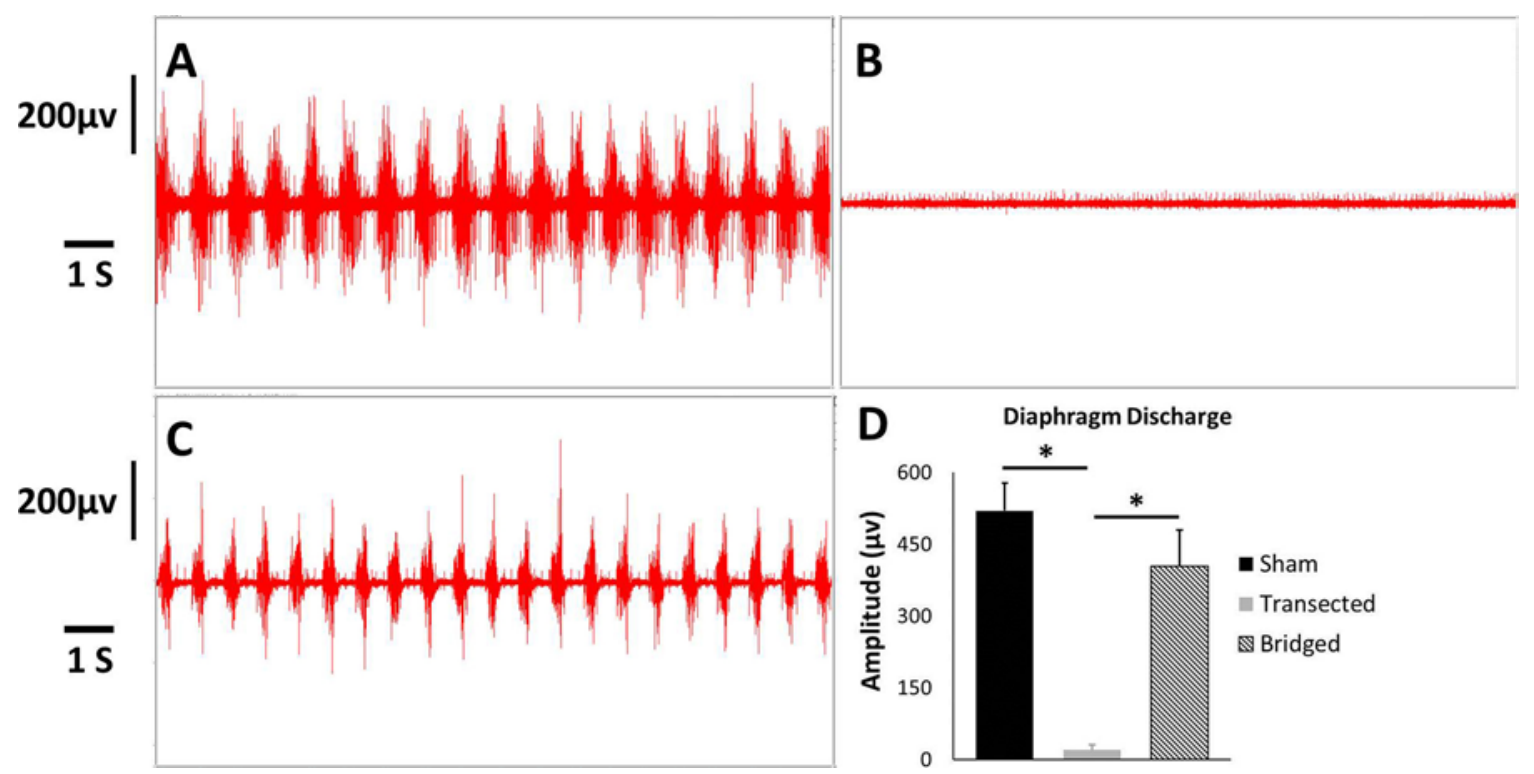

FIG. 8. Discharge of right diaphragm muscle in the sham group, transection group, and bridge group. A: Discharge of right diaphragm muscle in the sham group. Rhythmic and strong activities were recorded. B: Discharge of right diaphragm muscle in the transection group. No activity was noticed. C: Discharge of right diaphragm muscle in the bridge group. Rhythmic activities were recorded, even though the bursts were not as strong as in the sham group. D: Comparison of diaphragm muscle discharge in different groups. The error bars indicate standard deviations. ${ }^{*} p<0.0001$. Figure is available in color online only.

RLN and VN are motor fibers. We then demonstrated with morphological analyses that motor fibers from the RLN could regenerate into the PN and reinnervate the denervated diaphragm by end-to-end anastomosis in rabbits. Last, we demonstrated that RLN-PN anastomosis is an effective method in the restoration of rhythmic contraction of denervated diaphragm. Our findings suggest that the RLN is an optimal donor nerve to anastomose with PN to reestablish the autonomic movement of paralyzed diaphragm after high-level SCI.

\section{Acknowledgments}

This study was supported by the National Key Research and Development Program of China (2017YFA0105403).

\section{References}

1. Baldissera F, Cavallari P, Marini G, Tredici G: Diaphragm reinnervation by laryngeal motoneurons. J Appl Physiol (1985) 75:639-647, 1993

2. Bertelli J, Soldado F, Ghizoni MF, Rodríguez-Baeza A: Transfer of a terminal motor branch nerve to the flexor carpi ulnaris for triceps reinnervation: anatomical study and clinical cases. J Hand Surg Am 40:2229-2235, 2235.e1-2235.e2, 2015

3. Brown JO, Satinsky VP: Functional restoration of the paralyzed diaphragm following the cross-union of the vagus and phrenic nerves. Am J Med Sci 222:613-622, 1951

4. Calaresu FR, Pearce JW: Electrical activity of efferent vagal fibres and dorsal nucleus of the vagus during reflex bradycardia in the cat. J Physiol 176:228-240, 1965

5. Cederna PS: Discussion: a comparison of collateral sprouting of sensory and motor axons after end-to-side neurorrhaphy with and without the perineurial window. Plast Reconstr Surg 130:615-617, 2012
6. Chen LN, Zang WJ, Yu XJ, Liu J, Li DL, Kong SS, et al: Compensatory recovery of vagal control of hemodynamics after unilateral vagotomy. Physiol Res 57:119-132, 2008

7. Chhetri DK, Neubauer J, Sofer E: Posterior cricoarytenoid muscle dynamics in canines and humans. Laryngoscope 124:2363-2367, 2014

8. Derrey S, Verin E, Laquerrière A, de Barros AB, Lacoume Y, Fréger P, et al: Restoration of diaphragmatic function after diaphragm reinnervation by inferior laryngeal nerve; experimental study in rabbits. Respir Res 7:17-28, 2006

9. Duncan D, Eanes RH: Some results of substituting other nerves for the phrenic. Proc Soc Exp Biol Med 80:20-22, 1952

10. Evans DH, Murray JG: Histological and functional studies on the fibre composition of the vagus nerve of the rabbit. J Anat 88:320-337, 1954

11. Fewell JE, Zhang C, Gillis AM: Influence of adenosine $\mathrm{A}_{1^{-}}$ receptor blockade and vagotomy on the gasping and heart rate response to hypoxia in rats during early postnatal maturation. J Appl Physiol (1985) 103:1234-1241, 2007

12. Gauthier P, Baussart B, Stamegna JC, Tadié M, Vinit S: Diaphragm recovery by laryngeal innervation after bilateral phrenicotomy or complete $\mathrm{C} 2$ spinal section in rats. Neurobiol Dis 24:53-66, 2006

13. Guth L, Frank K: Restoration of diaphragmatic function following vagophrenic anastomosis in the rat. Exp Neurol 1:1-12, 1959

14. Haninec P, Kaiser R, Dubový P: A Comparison of collateral sprouting of sensory and motor axons after end-to-side neurorrhaphy with and without the perineurial window. Plast Reconstr Surg 130:609-614, 2012

15. Jiang BG, Yin XF, Zhang DY, Fu ZG, Zhang HB: Maximum number of collaterals developed by one axon during peripheral nerve regeneration and the influence of that number on reinnervation effects. Eur Neurol 58:12-20, 2007

16. Jiang Y, Gao B, Zhang X, Zhao J, Chen J, Zhang S, et al: Prevention and treatment of recurrent laryngeal nerve injury in thyroid surgery. Int J Clin Exp Med 7:101-107, 2014 
17. Karnovsky MJ, Roots L: "Direct-coloring" thiocholine method for cholinesterases. J Histochem Cytochem 12:219-221, 1964

18. Kobler JB, Datta S, Goyal RK, Benecchi EJ: Innervation of the larynx, pharynx, and upper esophageal sphincter of the rat. J Comp Neurol 349:129-147, 1994

19. Leiter JC, St-John WM: Phrenic, vagal and hypoglossal activities in rat: pre-inspiratory, inspiratory, expiratory components. Respir Physiol Neurobiol 142:115-126, 2004

20. Liao WC, Chen JR, Wang YJ, Tseng GF: The efficacy of endto-end and end-to-side nerve repair (neurorrhaphy) in the rat brachial plexus. J Anat 215:506-521, 2009

21. Morris AD, Lewis GM, Kucenas S: Perineurial glial plasticity and the role of TGF- $\beta$ in the development of the bloodnerve barrier. J Neurosci 37:4790-4807, 2017

22. Nandi S, Gutin G, Blackwood CA, Kamatkar NG, Lee KW, Fishell G, et al: FGF-dependent, context-driven role for FRS adapters in the early telencephalon. J Neurosci 37:56905698,2017

23. National Spinal Cord Injury Statistical Center: Spinal Cord Injury Facts and Figures at a Glance. Birmingham: University of Alabama at Birmingham, 2017 (https://www.nscisc. uab.edu/Public/Facts\%20and\%20Figures\%20-\%202017.pdf) [Accessed January 24, 2018]

24. Poliaček I, Stránsky A, Jakuš J, Baráni H, Tomori Z, Halasová E: Activity of the laryngeal abductor and adductor muscles during cough, expiration and aspiration reflexes in cats. Physiol Res 52:749-762, 2003

25. Steele AM, Gandhi MR, Sica AL: Phrenic and recurrent laryngeal motoneuron activities during hyperoxia and hypoxia in piglets. Brain Res Dev Brain Res 74:57-66, 1993

26. Tachikawa S, Nakayama K, Nakamura S, Mochizuki A, Iijima T, Inoue T: Coordinated respiratory motor activity in nerves innervating the upper airway muscles in rats. PLoS One 11:e0166436, 2016

27. Tomoda C, Hirokawa Y, Uruno T, Takamura Y, Ito Y, Miya A, et al: Sensitivity and specificity of intraoperative recurrent laryngeal nerve stimulation test for predicting vocal cord palsy after thyroid surgery. World J Surg 30:1230-1233, 2006

28. Tubbs RS, Pearson B, Loukas M, Shokouhi G, Shoja MM, Oakes WJ: Phrenic nerve neurotization utilizing the spinal accessory nerve: technical note with potential application in patients with high cervical quadriplegia. Childs Nerv Syst 24:1341-1344, 2008
29. Wang C, Yuan W, Zhou XH, Shi S, Wang X: Neurotization of the phrenic nerve with accessory nerve: a new strategy for high cervical spinal cord injury with respiratory distress. Med Hypotheses 76:564-566, 2011

30. Wen J, Yang M, Li L, Sun G, Tan J: Partial recovery of respiratory function and diaphragm reinnervation following unilateral vagus nerve to phrenic nerve anastomosis in rabbits. PLoS One 8:e79552, 2013

31. Yang ML, Li JJ, Zhang SC, Du LJ, Gao F, Li J, et al: Functional restoration of the paralyzed diaphragm in high cervical quadriplegia via phrenic nerve neurotization utilizing the functional spinal accessory nerve. J Neurosurg Spine 15:190-194, 2011

32. Yoshizawa H, Senda D, Natori Y, Tanaka R, Mizuno H, Hayashi A: End-to-side neurorrhaphy as Schwann cells provider to acellular nerve allograft and its suitable application. PLoS One 11:e0167507, 2016

33. Zakaria HM, Al Awad NA, Al Kreedes AS, Al-Mulhim AM, Al-Sharway MA, Hadi MA, et al: Recurrent laryngeal nerve injury in thyroid surgery. Oman Med J 26:34-38, 2011

\section{Disclosures}

The authors report no conflict of interest concerning the materials or methods used in this study or the findings specified in this paper.

\section{Author Contributions}

Conception and design: Tan, Sun, Zhang. Acquisition of data: Wen, Han, Guo, Wang, Hu, Liang, Wei, Zhou. Analysis and interpretation of data: Wen, Han, Guo, Yang, Li, Zhang. Drafting the article: Tan, Wen, Han, Guo. Critically revising the article: Tan, Zhang. Reviewed submitted version of manuscript: Tan, Wen, Han, Guo, Yang, Li, Zhang. Approved the final version of the manuscript on behalf of all authors: Tan. Statistical analysis: Yang, Li, Zhang. Administrative/technical/material support: Tan, Sun, Wang, Hu, Liang, Wei, Zhou, Zhang. Study supervision: Tan, Zhang.

\section{Correspondence}

Jun Tan: Shanghai East Hospital, Shanghai, China. dr.tan@139. com. 